\title{
INFLUÊNCIA DO MARKETING SOCIAL NA IMAGEM CORPORATIVA
}

\section{RESUMO}

Hoje, as empresas se preocupam em demonstrar afinidade com consumidores interessados em causas sociais. Estudos afirmam que houve aumento na responsabilidade social das empresas e que a atitude empresarial afeta a vontade de compra dos consumidores. Este artigo analisa em que medida o marketing social influencia na imagem corporativa, e busca identificar os principais fatores que influenciam na imagem corporativa das empresas que investem em marketing social e verificar a correlação entre os diferentes fatores que influenciam na imagem corporativa. Trata-se de estudo exploratório, quantitativo, pelo método levantamento de campo, com a aplicação de questionário autoadministrado e amostra de 220 universitários. Os dados foram analisados por meio de testes estatísticos de correlação de Spearman, Mann-Whitney e Wilcoxon W. Os resultados inferem correlação linear significativa entre marketing social e fatores que influenciam na imagem corporativa, mas não apontam correlação linear significativa entre os fatores que influenciam na imagem corporativa, investimento em marketing social e a frequência de uso de produtos. Este estudo apontou que marketing social é um fator de influência com baixa relevância na imagem corporativa, e que os fatores de influência mais importantes são qualidade e preço.

Palavras-chave: Campanha social; Imagem corporativa; Marketing relacionado a causas; Marketing social.

\begin{abstract}
Companies are investing to demonstrate an empathy with consumers interested in social issues. There was an increase in corporate social responsibility and some studies support that corporate attitude affects consumer purchasing. This paper aims to analyze to what extent social marketing campaigns influence the corporate image, also to identify the main factors that influence the corporate image of companies investing in social marketing and the correlation between the different factors that influence the corporate image. This is an exploratory study, quantitative, through a survey, with self-administered questionnaire to a sample of 220 college students. Data were analyzed using statistical tests of Spearman Correlation, Mann-Whitney and Wilcoxon W. The results infer significant linear correlation between social marketing campaigns and factors that influence the corporate image, however there is not a significant linear correlation between the factors that influence the corporate image, investment in social marketing and the frequency of use of the products. The study found that social marketing campaigns is a factor influencing the corporate image with low relevance, and that the most important influence factors are the quality and price of products.
\end{abstract}

INFLUENCE OF SOCIAL MARKETING IN CORPORATE IMAGE

Keywords: Social Marketing; Cause Related Marketing; Corporate Image; Social Campaign. 


\section{INFLUENCIA DEL MARKETING SOCIAL EN LA IMAGEN CORPORATIVA}

\section{RESUMEN}

Las empresas están invirtiendo para demostrar una empatía con los consumidores interesados en las cuestiones sociales. Hubo un aumento en la responsabilidad social de las empresas y algunos estudios apoyan que la actitud corporativa afecta el poder adquisitivo de los consumidores. En este trabajo se pretende analizar en qué medida las campañas de marketing social influyen en la imagen corporativa, además de identificar los principales factores que influyen en la imagen corporativa de las empresas que invierten en marketing social y la correlación que existe entre los diferentes factores que influyen en la imagen corporativa. Se trata de un estudio exploratorio, cuantitativo, a través de una encuesta, con el auto-administrado cuestionario a una muestra de 220 estudiantes universitarios. Los datos fueron analizados mediante pruebas estadísticas de correlación de Spearman, MannWhitney y Wilcoxon W. Los resultados infieren correlación lineal significativa entre las campañas y los factores que influyen en la imagen corporativa de mercadeo social, sin embargo no hay una correlación lineal significativa entre los factores que influyen en la empresa imagen, la inversión en marketing social y la frecuencia de uso de los productos. El estudio encontró que las campañas de marketing social son un factor que influyen en la imagen corporativa con baja relevancia, y que los factores de influencia más importantes son la calidad y precio de los productos.

Palabras clave: Marketing Social; Causa marketing relacionados; Imagen corporativa; Campaña Social.

\footnotetext{
${ }^{1}$ Doutora em Saúde Pública pela Universidade de São Paulo - USP. Professora do Programa de Mestrado Profissional em Gestão em Sistemas de Saúde da Universidade Nove de Julho - PMP-GSS/Uninove. Brasil. Email: soniamonken@uninove.br

${ }^{2}$ Doutor em Administração pela Universidade de São Paulo - USP. Professor do Programa de Pós-Graduação Mestrado em Administração da Universidade do Sul de Santa Catarina - Unisul. Brasil. E-mail: alessio.sarquis@ig.com.br

${ }^{3}$ Doutor em Administração pela Universidade do Vale do Rio dos Sinos - Unisinos. Professor do Programa de Pós-Graduação Mestrado em Administração da Universidade de Caxias do Sul - UCS. Brasil. E-mail: vtondolo@gmail.com

${ }^{4}$ Doutor em Engenharia de Produção pela Universidade Federal de Santa Catarina - UFSC. Professor da Universidade do Sul de Santa Catarina - Unisul. Brasil. E-mail: mauricio.lima@unisul.br
} 


\section{INTRODUÇÃ̃O}

Nas últimas décadas, estudos têm revelado que há empresas preocupadas em demonstrar afinidade com consumidores que se interessam por esporte, evento e causas sociais (Brunk, 2010; Mejri \& De Wolf, 2012; Nascimento, Gimenez \& Campomar, 2014). No passado, as empresas patrocinavam atividades sociais simplesmente por decisão pessoal ou grupo de executivos. Hoje, os investimentos em causas sociais estão cada vez mais alinhados com os objetivos e as estratégias da organização (Gimenez \& Campomar, 2014).

Programas de marketing social são projetados para atingir objetivos, tais como melhoria da reputação corporativa, diferenciação da marca, atração de novos consumidores, lealdade à marca, manutenção de funcionários e para o aumento da lucratividade ou preços de ações (Lee \& Kotler, 2011; Bloom, Hoeffler, Keller \& Meza, 2012; Carrol \& Buchholtz, 2014). No entanto, há dúvidas se as empresas estão atingindo esses objetivos. Há evidências de que o investimento em esporte, entretenimento e causas sociais pode ser bem-sucedido, mas pesquisa empírica sobre o tema é, ainda, limitada. Há, inclusive, necessidade de compreender melhor os efeitos do marketing social na imagem corporativa (Bloom et al., 2012).

Os estudos sobre influência do marketing social na imagem corporativa têm levantado diversas controvérsias (Vieira, Higuchi, Oliveira \& Correia, 2007; Moretti \& Figueiredo, 2008; Silva, Mota \& Mota, 2014). Para alguns autores, a responsabilidade social corporativa (RSC) é entendida como um posicionamento ético da empresa em relação à sociedade, visando atender anseios filantrópicos e comunitários, bem como a demanda de stakeholders relevantes (Vieira et al., 2007). Outros autores a consideram como atividades realizadas com propósito de promover valores sociais e satisfazer necessidades da sociedade (Wu \& Wang, 2014). Além disso, somase o fato de que as empresas estão sendo mais conhecidas pelos consumidores, e que o cidadão está mais consciente que a atitude empresarial pode influenciar na decisão de compra dos produtos (Brunk, 2010; Mejri \& De Wolf, 2012).

No Brasil, as pesquisas sobre marketing social indicam que as empresas têm assumido cada vez mais responsabilidade com a comunidade onde estão inseridas (Ikeda et al., 2000; Camargo, 2001; Vieira et al., 2007; Novais et al., 2010; Albino et al, 2013). Em 1998, o Instituto Ethos de Empresas e Responsabilidade Social foi criado com propósito de mobilizar, sensibilizar e apoiar as empresas interessadas em dispor de gestão sustentável e socialmente responsável. Segundo Ethos (2015), o faturamento de seus associados representa $35 \%$ do PIB brasileiro e reúne 586 empresas de diferentes setores/ramos de atividades, e das diversas regiões/estados do Brasil. Desse universo, 13,6\% são microempresas; 22,5\%, pequenas empresas; $19,2 \%$, médias empresas e 41,6\%, empresas de grande porte.

Diante disso, decidiu-se pela realização deste estudo com o seguinte problema de pesquisa: Em que medida campanhas de marketing social influenciam na imagem corporativa? Assim, o objetivo desse estudo é analisar em que medida campanhas de marketing social influenciam na imagem corporativa, na perspectiva do público alunos universitários. Especificamente, este estudo busca identificar as principais empresas que investem no Brasil em marketing social e a frequência de uso de seus produtos; levantar os fatores que influenciam na imagem corporativa das empresas que investem em marketing social; verificar a correlação entre fatores que influenciam na imagem corporativa e a frequência de uso dos produtos; verificar a correlação entre os diferentes fatores que influenciam na imagem corporativa das empresas que investem em marketing social.

Este estudo contribui para ampliar o conhecimento acadêmico sobre a influência do marketing social na imagem corporativa, bem como auxiliar futuros estudos acadêmicos sobre o tema, seja na formulação de hipóteses de pesquisa e/ou no direcionamento para futuros estudos. Essa pesquisa contribui, também, para verificar algumas proposições teóricas presentes na literatura, no tocante aos fatores de influência do marketing social (Lee \& Kotler, 2011), e para confirmar resultados de outros estudos já realizados (Novais et al., 2010; Da Silva et al., 2012; Pozo \& Tachizawa, 2012; Froemming \& Souza, 2013; Patino et al., 2014).

Esse artigo contém uma revisão teórica dos conceitos de marketing social e imagem corporativa, depois a descrição do método de pesquisa e a apresentação/discussão dos resultados. Ao final, contém as considerações finais, com limitações e sugestões para futuros estudos sobre o tema.

\section{FUNDAMENTAÇÃO TEÓRICA}

\subsection{Panorama sobre o marketing social}

Segundo Nomura e De Souza (2007), entender na literatura a atuação do marketing no campo social é, ainda, um desafio. Os estudiosos não conseguiram ainda chegar a uma concepção 
menos extremista sobre o tema. A expressão marketing social apareceu pela primeira vez em 1971 e foi utilizada para "[...]descrever o uso de princípios e técnicas de marketing para a promoção de uma causa, ideia ou comportamento social" (Kotler \& Roberto, 1992, p.25). Estes autores definiram marketing social como "[...]uma tecnologia de administração de mudança social, associada ao projeto, implantação e ao controle de programas voltados para o aumento da disposição de aceitação de uma ideia ou prática social em um ou mais grupos adotantes escolhidos como alvos".

Segundo Pringle e Thompson (2000), o marketing social é direcionado para promover mudanças de valores e comportamentos de indivíduos ou grupos, não enfatizando questões sobre necessidades e desejos de consumidores, nem tampouco produtos, mercados e empresas com fins lucrativos. Para estes autores, as ações sociais das empresas não utilizam, na maioria das vezes, estratégias de marketing social, mas a promoção social por meio de estratégias de marketing tradicional. Wilmer e Basi (2007) e Lee e Kotler (2011) entendem marketing social como o uso de princípios e técnicas de marketing para influenciar um grupo de clientes a aceitar ou rejeitar voluntariamente determinado comportamento em benefício da sociedade.

De acordo com Nomura e De Souza (2007), o marketing social busca encorajar e transferir para indivíduos e/ou grupos modelos de promoção do bem-estar social, criar novas opiniões e emoções, e promover mudança comportamental em relação a questões sociais, garantindo uma efetividade na responsabilidade social, individual e coletiva. De fato, as atividades de marketing social têm foco no interesse público, busca manter o bemestar social e desenvolver produtos respeitando as exigências da sociedade. Apesar dos diferentes conceitos e visões sobre o tema, o marketing social tem enfoque similar ao marketing tradicional (ou seja, preocupado com aspectos de consumo e mercado), mas procura gerar benefícios para a sociedade e para o meio-ambiente, inclusive para a empresa e seus consumidores (Brenkert, 2002).

Ikeda et al. (2000) afirmam que o estabelecimento de estratégias de marketing que atendam as necessidades dos consumidores e das empresas é, cada vez mais, um desafio para as organizações. Os consumidores estão mais criteriosos na escolha de marcas/produtos e passaram a valorizar produtos de empresas que contribuem efetivamente com a sociedade. Diante disso, conforme Kotler e Fox (1994), as estratégias de marketing precisam atender a três demandas importantes, e, eventualmente, conflitantes: lucro da empresa, satisfação dos consumidores e interesse público.

Andreasen (1991) apontou que estratégias do marketing social são utilizadas com propósito de criar consciência e interesse dos consumidores em determinada atitude ou comportamento relacionado a situações de compra. Exemplos de estratégias de marketing social utilizadas são: mudança de valores na qualidade de vida dos consumidores, sensibilização para atitudes que façam diferença no consumo de produtos, ações de pós-venda para apoiar um novo comportamento de consumo e agregação de valores sociais aos consumidores.

Nomura e De Souza (2007) afirmaram que o marketing social no Brasil vem sendo utilizado com maior frequência por empresas privadas e organizações do terceiro setor, e que esse termo é usado, na maioria das vezes, para designar estratégias de comunicação e divulgação. Já Weinreich (2011) aponta como exemplos equivocados de estratégias de marketing social os seguintes: desenvolvimento de ações para angariar fundos, ações de comunicação/promoção com propósito exclusivo de divulgar ações sociais realizadas e desenvolver campanhas sociais visando apenas desenvolver a imagem corporativa junto a determinado público-alvo.

\subsection{Campanhas de marketing social e a imagem corporativa}

A imagem é um conjunto de suposições, atitudes e impressões que indivíduos ou grupos têm acerca de determinado produto, marca ou organização, neste caso denominado imagem corporativa (Rabaça, 1996; Kotler \& Barich, 2001). A imagem corporativa pode compreender percepções e atitudes dos stakeholders sobre ações, atividades e relacionamentos da organização (Nascimento, Jimenez \& Campomar, 2014), visto que consumidores e empresas estabelecem relações entre si e buscam relações sociais.

Segundo Tavares (1998, p.65), “[...]as relações da empresa com seu público e a forma como esta trata as questões ambientais e sociais ocorrem em vários níveis, que correspondem às várias perspectivas de imagem". No primeiro nível, a imagem é formada a partir da maneira como a empresa delineia sua estratégia e estabelece suas políticas de produto, preço, distribuição e promoção. No segundo nível, a imagem compreende impressões deixadas por empregados, vendedores e aparência de escritório, fábrica e instalações. No terceiro nível, compreende as consequências das relações com vários públicos, incluindo imprensa, formadores de opinião, comunidade, fornecedores, intermediários e 
concorrentes. No quarto nível, a imagem é afetada por fatores provenientes do setor de negócios e do contexto em que a organização está inserida. Estes fatores do setor/mercado estão fora do controle da empresa e incluem questões como aspectos políticos, econômicos, concorrenciais e sociais.

Conhecer como se forma a imagem corporativa ajuda a entender melhor os fatores que afetam a imagem da organização e a compreender como promover mudanças desejadas. Kotler (1978, p. 150) acredita que "[...]uma imagem é tanto influenciada pelas características do objeto e como pelas características subjetivas daquele que as está percebendo". Já Grönroos (1995), afirmou que uma imagem corporativa positiva e bem conhecida é um patrimônio valioso para qualquer empresa, pois causa impacto na percepção dos consumidores e do mercado. Assim, a imagem corporativa consiste em um conjunto de suposições, atitudes e impressões que um indivíduo ou grupo tem acerca de uma organização.

De acordo com Kotler e Roberto (1992), uma campanha de marketing social é um esforço organizado feito pela organização ou determinado grupo (agentes de mudança) com propósito de convencer terceiros (adotantes escolhidos como alvo) a aceitar, modificar ou abandonar certas atitudes, ideias e/ou comportamentos. Segundo estes autores, as campanhas sociais surgiram na história antiga e envolvem, atualmente, um conjunto de ações direcionadas para promover melhorias na saúde (ex: contra fumo e drogas), na preservação ambiental (água e florestas), na reforma educacional (alfabetização e escolas públicas) e no ambiente econômico (atração de investidores estrangeiros), dentre outras.

Algumas deficiências têm sido apontadas nas campanhas de marketing social (Kotler \& Roberto, 1992). Essas campanhas carecem de monopolização, canalização e suplementação de esforços; às vezes, são apenas ações de comunicação (informacional) e feitas sem planejamento adequado. Precisam ser pré-testadas, planejadas com a participação do público-alvo e alinhadas com a estratégia de Responsabilidade Social Corporativa (RSC) da organização. A Responsabilidade Social Corporativa envolve a identificação, antecipação e gerenciamento das expectativas dos diversos grupos da sociedade ligados, direta ou indiretamente, à organização (Vieira et al., 2007; Maon, Lindgreen \& Swaen, 2010).

Para incorporar valor social à imagem corporativa, as empresas investem em contribuições sociais, conduta adequada em relação aos empregados, inovação nos negócios e/ou no relacionamento com a sociedade, credibilidade da marca, confiabilidade da força de vendas, qualidade/reparabilidade de produtos, iniciativas de educação, e zelam pela conduta ética em relação à definição de preços, descontos e financiamentos (Kotler \& Barich, 2001). No entanto, campanhas que oferecem benefícios sociais são, muitas vezes, consideradas, apenas, como custo, dependem do compromisso da direção/proprietários e oscilam conforme a situação/rentabilidade da organização (Vieira et al., 2007; Maon, Lindgreen \& Swaen, 2010). Atualmente, em nível mundial, empresas, tais como Procter \& Gamble, BMW, Nestlé e China Vanke Co. Ltd, têm desenvolvido campanhas de marketing social que geram tanto benefícios públicos quanto resultados financeiros para a organização (Kloppenborg \& Tesch, 2015).

Kloppenborg e Tesch (2015) analisaram relatórios corporativos de responsabilidade social de várias empresas que investem em projetos sociais e entrevistaram executivos de vários níveis hierárquicos sobre a política de investimento em projetos sociais da organização. Os resultados apontam algumas estratégias comuns nessas empresas. Primeiro, a criação de mecanismos para obter a adesão de diferentes parceiros da sociedade, incluindo reunião de atores com diferentes interesses, percepções e capacidades, e a participação de acadêmicos, grupos de clientes e instituições públicas. Segundo, investir em incubadoras comprometidas com a criação de valor social e na integração entre a iniciativa privada e o poder público. Terceiro, a maioria das empresas analisadas construiu parcerias com propósito de fortalecer a capacidade de execução e introdução de novos projetos sociais.

O Meijri e De Wolf (2012) destacaram também que fatores que impactam na imagem corporativa são aspectos econômicos (ex: preços e forma de pagamento), sociais e ambientais. A análise de relatórios corporativos de responsabilidade social das empresas analisadas revelou que, para comunicar ações/iniciativas sociais, as empresas adotam um estilo de narrativa consubstanciado pelo uso frequente de verbos ativos e adjetivos objetivos, e que evitam o discurso de propaganda com base em julgamentos subjetivos e promessas.

Conforme Kloppenborg e Tesch (2015), a criação de parcerias com valor sinérgico requer, no entanto, algumas necessidades especiais. Muitas vezes, a empresa não tem capacidades específicas ou legitimidade para obter as parcerias necessárias e precisa ser aceita pelo público-alvo da campanha. Elas podem também atingir grupos de clientes que podem não encontrar nas marcas da empresa a imagem corporativa desejada, nem canais de distribuição atraentes. Além disso, na parceria com 
instituições governamentais ou organizações nãogovernamentais, a empresa pode não encontrar a legitimidade e/ou credibilidade política necessária para ser bem-sucedida junto ao mercado. Empresas que se esforçam para controlar o processo de criação de valor e a sinergia social complementam os seus sistemas de controle com perspectivas de diferentes partes/agentes interessados. Sistemas de monitoramento de múltiplas perspectivas possibilitam medir o valor social da organização e fornecer uma base importante para a criação de valor sinérgico (Kloppenborg e Tesch, 2015). Essa sinergia pode estimular o surgimento de novos produtos e aumentar a participação de mercado e lucratividade da organização, além da imagem da empresa perante clientes, acionistas e outros grupos sociais.

De Oliveira (2006) analisou 231 empresas brasileiras filiadas ao Instituto Ethos de Responsabilidade Social e concluiu que apenas $19,7 \%$ delas utilizam efetivamente estratégias de marketing social. Este estudo apontou também que as estratégias mais utilizadas são: construção de nova consciência, mudança de comportamento, consumo consciente, envolvimento da comunidade e motivação de fornecedores para práticas sociais responsáveis. Apontou também que ações voltadas para o consumo consciente estão mais presentes nas organizações que se preocupam com o impacto da produção industrial, com a qualidade dos produtos e com a relação com a sociedade. E que as ações de envolvimento da comunidade estão mais presentes nas organizações que investem em programas ambientais, educacionais e de geração de renda.

Silva e Chauvel (2011) investigaram o comportamento do consumidor em relação ao tema por meio de pesquisa qualitativa e entrevistas em profundidade com profissionais de engenharia e empreendedores. Os resultados apontam que os entrevistados percebem o tema como importante, mas têm diferentes percepções sobre o assunto. Muitos deles não se lembram de ter comprado produtos de empresas socialmente responsáveis e alegam falta de informação, pressa, ausência de selo de garantia e que valorizam o preço e a qualidade na decisão de compra. Os depoimentos indicam também que há necessidade de maior divulgação das ações sociais pelos fabricantes e de selo de garantia social, algo que poderia reduzir o ceticismo e incentivar a responsabilidade social de empresas e consumidores.

De acordo com Mazutis e Slawinski (2015), dois fatores caracterizam a autenticidade das campanhas de marketing social da organização: a distinção e a conexão. A distinção mensura a intensidade com que as atividades de uma empresa estão alinhadas com a sua missão, visão e seus valores; enquanto a conexão refere-se ao grau de incorporação dos esforços em um contexto social mais amplo. Essas duas dimensões afetam a percepção dos consumidores e podem ajudar na construção da imagem corporativa. Além disso, políticas, sistemas de gestão e programas organizacionais podem estimular a sinergia na prática ambiental e social nos demais membros da cadeia de suprimento da organização. Nesse sentido, Meijri e De Wolf (2012) afirmaram que as empresas varejistas têm se esforçado mais para demonstrar comportamentos socialmente responsáveis.

$\mathrm{Na}$ perspectiva da imagem corporativa, Brunk (2010) identificou seis domínios e 36 subdomínios no comportamento social das empresas que causam impacto em diferentes públicos (consumidores, empregados, comunidade exterior, economia local e comunidade empresarial). Os principais domínios identificados são: serviços agregados, qualidade percebida, preço dos produtos, inovação de produtos/serviços e marketing social. Os resultados de Brunk (2010) sugerem também que há disparidade entre as perspectivas do consumidor e a dos negócios, e que as fontes de consumo ético que afetam a imagem corporativa são mais diversificadas e complexas do que apresenta a literatura do tema.

\section{MÉTODO DE PESQUISA}

Este estudo classifica-se como exploratório, de abordagem quantitativa e na forma de levantamento de campo (survey). Devido ao pouco conhecimento acerca da influência do marketing social na imagem corporativa, como apontado por Lee e Kotler (2011) e Moretti e Figueiredo (2008), decidiu-se pela escolha de um estudo exploratório e para analisar em que medida campanhas de marketing social influenciam na imagem corporativa, na perspectiva do público alunos universitários. Conforme Collis e Hussey (2005), estudos exploratórios são recomendados quando há pouco conhecimento sobre o problema investigado e/ou quando se deseja procurar padrões, ideias ou hipóteses a serem testadas. Para Richardson (2008), a abordagem quantitativa caracteriza-se pelo emprego da quantificação na coleta e/ou no tratamento de dados e pelo uso de técnicas estatísticas de análise.

No âmbito deste estudo, o público aluno universitário compreende todas as pessoas residentes na região metropolitana de São Paulo/SP (Brasil) e com formação universitária em andamento. Neste grupo, enquadram-se as pessoas que estejam cursando uma universidade em nível de 
graduação e pós-graduação em nível de especialização ou stricto sensu. A amostragem do estudo é do tipo não-probabilística, por conveniência (aqueles que aceitaram participar do estudo). A amostra foi constituída por 338 alunos universitários provenientes da Universidade Mackenzie, Universidade de São Paulo (USP) e Universidade Nove de Julho (Uninove). Segundo o Sindicato das Mantenedoras de Estabelecimentos de Ensino Superior no Estado de São Paulo (Simesp, 2010), a região metropolitana de São Paulo/SP e a região de Campinas/SP concentram a maior parte das matrículas em cursos presenciais das redes pública e privada de ensino superior no Estado de São Paulo, e respondem por 1,06 milhão de matrículas, ou seja, $71,3 \%$ do total alunos universitários do Estado de São Paulo.

Como método de coleta de dados, na pesquisa de campo foi utilizado questionário autoadministrado (preenchido pelos próprios alunos pesquisados), com perguntas fechadas e elaborado com base nos estudos de Brunk (2010) sobre comportamento ético de marcas e de Mejri e De Wolf (2012), da lista de adjetivos identificados na análise de relatórios de responsabilidade social e sustentabilidade de empresas varejistas. $\mathrm{O}$ questionário contém perguntas sobre perfil dos participantes, empresas que investem no Brasil em marketing social, frequência de uso de seus produtos e fatores que influenciam na imagem corporativa. Os fatores de influência da imagem foram mensurados por meio de uma escala de 5 pontos (onde $0=$ sem influência; $5=$ elevada influência) e pré-testados com uma amostra de dez alunos do curso de Administração da Universidade Mackenzie. A coleta de dados ocorreu no ambiente das universidades pesquisadas em agosto/setembro de 2013.

Os dados coletados foram tabulados por meio do software SPSS versão Windows 10 . No tratamento dos dados, além descrição das variáveis envolvidas no estudo (média aritmética, desviopadrão, contagem e percentual), foram utilizadas as técnicas estatística Análise de Correlações Ordinais, baseada no Coeficiente de Correlação Ordinal de Spearman, e Análise de Correlação Bisserial de Pontos, baseada no Teste de Mann-Whitney e Wilcoxon W para amostras independentes na análise da correlação entre variáveis. Em todas as análises de testes estatísticos realizadas, o $p$-value foi calculado para medir a probabilidade do valor observado para a estatística de teste ser casual. Sempre que o resultado do teste de correlação foi menor que $5 \%(\mathrm{p}<0,05)$, decidiu-se por considerar a correlação estatisticamente significativa, como recomendam Hair et al. (2009).

\section{APRESENTAÇÃO E ANÁLISE DOS DADOS}

Esta seção apresenta os resultados e as análises realizadas dos dados coletados na pesquisa de campo desse estudo. De início, contém os resultados de empresas que investem em marketing social e a frequência de uso de seus produtos; depois os resultados de fatores que influenciam na imagem corporativa das empresas e, em seguida, os resultados da análise estatística da correlação entre variáveis. No primeiro teste estatístico, a variável dependente é a frequência de uso dos produtos e as variáveis independentes são fatores que influenciam na imagem corporativa. No segundo teste estatístico, foi feita a análise de correlação entre os diferentes fatores que influenciam na imagem corporativa. Ao final, contém a discussão dos resultados.

Quanto ao perfil dos participantes, a amostra é composta por alunos universitários do município de São Paulo/SC, Brasil, da Universidade Mackenzie, Universidade de São Paulo e Universidade Nove de Julho $(\mathrm{n}=338)$, sendo $44,7 \%$ pessoas do sexo masculino e $55,3 \%$, do sexo masculino; $73 \%$ deles possuem idade inferior a 25 anos, caracterizando um público de perfil predominantemente jovem. A grande maioria dos participantes (superior a 2/3 da amostra) pertence também à classe social média-alta, com renda média familiar mensal entre $\mathrm{R} \$ 8.137,00 \mathrm{e}$ $\mathrm{R} \$ 12.204,00$.

\subsection{Resultados de empresas que investem em marketing social e uso de produtos}

Um dos objetivos deste estudo é identificar as principais empresas que investem em marketing social no Brasil e a frequência de uso de seus produtos pelos alunos universitários pesquisados. Para tanto, os participantes do estudo foram indagados sobre quais empresas, dentre as que conhecem, investem no Brasil em campanhas de marketing social, se utilizam produtos dessas empresas e qual a frequência de uso, e se a prática de marketing social das empresas é considerada quando da decisão de compra dos produtos.

A Tabela 1 apresenta os resultados das empresas que investem no Brasil em campanhas de marketing social, na opinião dos alunos universitários pesquisados $(\mathrm{n}=338)$. A grande maioria dos participantes $(66,3 \%$ deles) assinalou que conhece empresas que investem em marketing social no Brasil e as empresas mais mencionadas são: Nestlé (44\% de citação), Procter \& Gamble (9\%), Grupo Fleury (6\%), MSF do Brasil (5\%) e outros $(36 \%)$, com várias empresas citadas, incluindo Fiat Automóveis, Natura Cosméticos, 
Fundação Bradesco, McDonald e Lojas C\&A Modas, por exemplo. Entretanto, um percentual significativo dos alunos pesquisados $(33,7 \%)$ declarou não conhecer quaisquer empresas no Brasil que invistam em campanhas de marketing social.

Tabela 1 - Resultados de empresas que investem em marketing social

\begin{tabular}{|c|c|c|c|}
\hline \multirow{2}{*}{ CONHECIMENTO } & CONTAGEM & $\boldsymbol{\%}$ & \% ACUMULADO \\
\hline Não & 114 & 33,7 & 33,7 \\
\hline Sim & 224 & 66,3 & 100,0 \\
\hline Total & 338 & 100,0 & \\
\hline
\end{tabular}

Fonte: Dados da pesquisa (2013)

A Tabela 2 apresenta os resultados de uso de produtos das empresas que investem no Brasil em marketing social. Do total de 220 alunos pesquisados, a grande maioria deles (ou seja, 73,2\% da amostra) declarou que utiliza produtos de empresas que investem em marketing social, mas $26, \%$ declarou que não utiliza produtos destas empresas. Dentre os que declararam utilizar produtos destas empresas, 49,3\% mencionou que utiliza frequentemente os produtos; $39,7 \%$ disse que utiliza apenas periodicamente e $10 \%$ que utiliza raramente. Os alunos classificaram como frequentemente quando o produto é utilizado pelo menos uma vez por semana; periodicamente quando o produto é utilizado, pelo menos, uma vez por mês; raramente quando o produto é utilizado de forma mais ocasional ou esporádica.

Tabela 2 - Resultados de uso de produtos e frequência de uso pelos alunos pesquisados

\begin{tabular}{|c|c|c|c|}
\hline USO DO PRODUTO & CONTAGEM & \% & \% ACUMULADO \\
\hline Não & 59 & 26,8 & 26,8 \\
\hline Sim & 161 & 73,2 & 100,0 \\
\hline Total & 220 & 100,0 & \% ACUMULADO \\
\hline FREQUÊNCIA DE USO & CONTAGEM & $\%$ & 10,0 \\
\hline Raramente & 20 & 10,0 & 49,7 \\
\hline Periodicamente & 77 & 39,7 & 100,0 \\
\hline
\end{tabular}

Fonte: Dados da pesquisa (2013)

A Tabela 3 mostra os resultados da quantidade de alunos universitários que levam em consideração a prática de marketing social da empresa quando da decisão de compra de novos produtos. Do total de participantes $(n=315$ respondentes), 74,3\% deles declararam que levam em consideração a prática de marketing social da empresa e $25,7 \%$ declaram não levam isto em consideração no momento da decisão de compra. 
Tabela 3 - Quantidade de alunos que adquiriram produtos das empresas

\begin{tabular}{|c|c|c|c|}
\hline COMPRA & FREQ & \% & \% ACUMULADO \\
\hline Não & 81 & 25,7 & 25,7 \\
\hline Sim & 234 & 74,3 & 100,0 \\
\hline Total & 315 & 100,0 & \\
\hline
\end{tabular}

Fonte: Dados da pesquisa (2013)

\subsection{Resultados de fatores que influenciam na imagem corporativa}

Um objetivo específico deste estudo é levantar os fatores que influenciam na imagem corporativa das empresas que investem em marketing social. Com base em uma lista de fatores, os alunos foram solicitados a avaliarem aqueles que, na sua opinião, mais influenciam na imagem corporativa das empresas que investem em marketing social. A Figura 2 apresenta os resultados de fatores assinalados, incluindo a média aritmética e o desvio-padrão $(\mathrm{n}=338)$. Com média 5 , os dois fatores de influência avaliados com elevada intensidade são: qualidade dos produtos e preço de venda dos produtos. Com média 3, o fator de influência considerado menos relevante é marketing social. E os demais fatores (exposição na mídia, ética nos negócios, serviço de pós-venda, variedade de produtos, inovação nos produtos, condições de pagamento e facilidade de acesso), todos com média 4, foram considerados como de influência moderada na imagem corporativa. Todos dos fatores mensuradas apresentam resultados de desvio-padrão baixo (inferior a $20 \%$ da escala aplicada, como recomendam Hair et al., 2009), indicando baixa dispersão dos valores em relação à média e baixa variabilidade na opinião dos alunos universitários pesquisados.

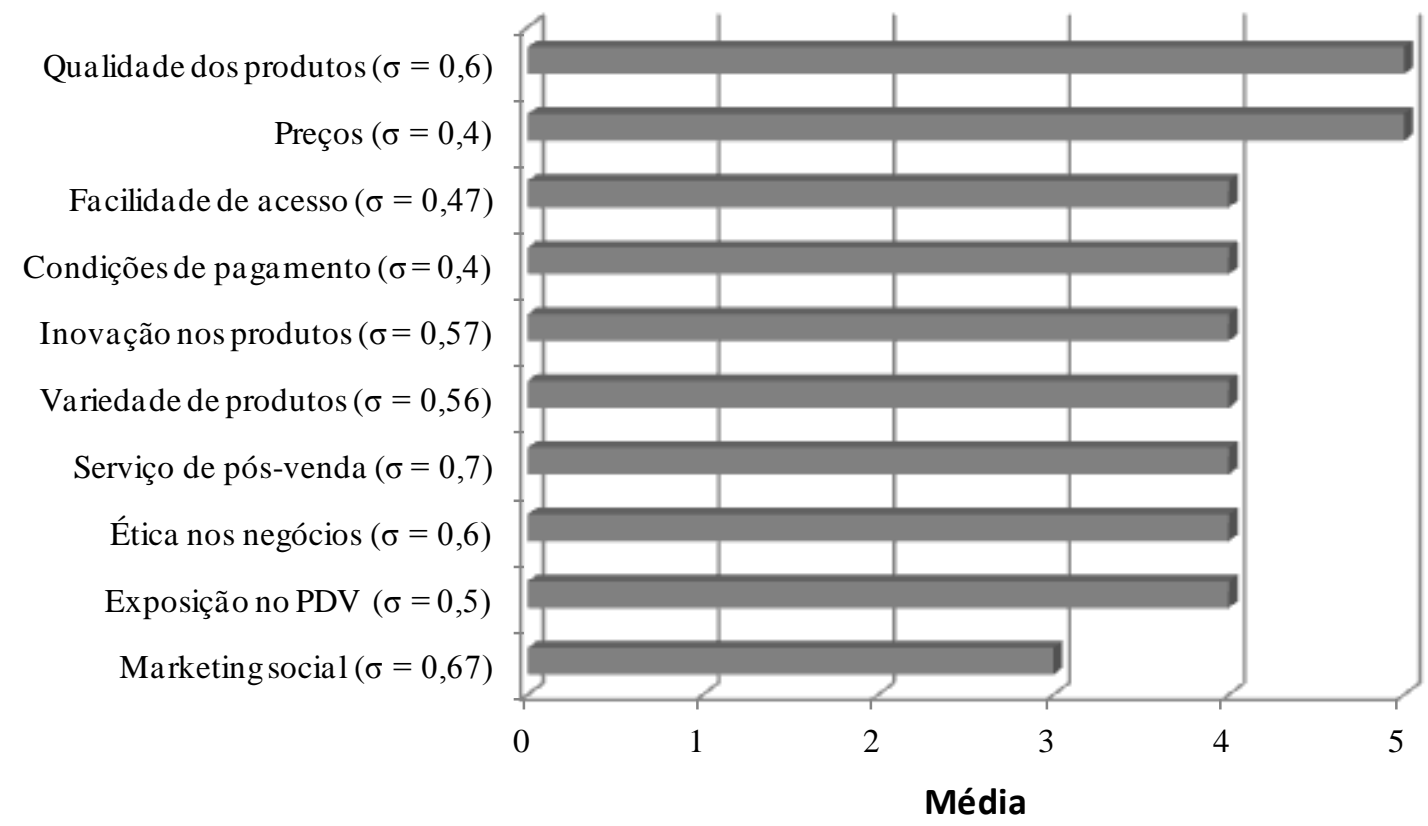

Figura 2 - Resultados de fatores que influenciam na imagem corporativa $(n=338)$

Fonte: Dados da pesquisa (2013)

\subsection{Resultados da análise estatística da correlação entre variáveis}

De início, a Tabela 4 apresenta os resultados das medidas descritivas dos fatores que influenciam na imagem corporativa por frequência de uso pelos alunos pesquisados (amostra varia de 193 e 194 participantes). Nesta análise, dois grupos de alunos foram constituídos conforme a frequência de uso dos produtos das empresas que investem em 
marketing social. O grupo 1 contém 20 participantes, aqueles que mencionaram utilizar apenas raramente ou periodicamente os produtos de empresas. O grupo 2 contém 174 participantes, aqueles que declararam o uso frequente de produtos das empresas. Os resultados da tabela mostram a quantidade de participantes, os valores de postos médios para ambos os grupos e a soma dos postos por fator de influência na imagem corporativa. Postos são posições, em números, que os fatores ocupam quando colocados em ordem crescente de citação (Hair et al., 2009). Os fatores com posto médio mais elevados são: inovação (média do posto nos grupos: 102,2), condições de pagamento $(100,2)$, facilidade de acesso $(98,3)$ e exposição no PDV $(100,1)$.

Tabela 4 - Medidas descritivas dos fatores de influência na imagem corporativa

\begin{tabular}{|c|c|c|c|c|}
\hline Item & $\begin{array}{c}\text { Freq. de } \\
\text { uso }\end{array}$ & $\mathbf{N}$ & $\begin{array}{l}\text { Posto } \\
\text { Médio }\end{array}$ & $\begin{array}{c}\text { Soma de } \\
\text { Postos }\end{array}$ \\
\hline \multirow{2}{*}{$\begin{array}{l}\text { Serviço pós-venda } \\
(\mathrm{n}=193)\end{array}$} & 1 & 20 & 92,53 & 1850,5 \\
\hline & 2 & 173 & 97,52 & 16870,5 \\
\hline \multirow{2}{*}{$\begin{array}{c}\text { Variedade } \\
(\mathrm{n}=194)\end{array}$} & 1 & 20 & 95,2 & 1904 \\
\hline & 2 & 174 & 97,76 & 17011 \\
\hline \multirow{2}{*}{$\begin{array}{c}\text { Preço } \\
(\mathrm{n}=194)\end{array}$} & 1 & 20 & 95,8 & 1916 \\
\hline & 2 & 174 & 97,7 & 16999 \\
\hline \multirow{2}{*}{$\begin{array}{c}\text { Ética } \\
(\mathrm{n}=194)\end{array}$} & 1 & 20 & 97 & 1940 \\
\hline & 2 & 174 & 97,56 & 16975 \\
\hline \multirow{2}{*}{$\begin{array}{l}\text { Marketing social } \\
\quad(\mathrm{n}=194)\end{array}$} & 1 & 20 & 94,63 & 1892,5 \\
\hline & 2 & 174 & 97,83 & 17022,5 \\
\hline \multirow{2}{*}{$\begin{array}{c}\text { Qualidade } \\
(\mathrm{n}=194)\end{array}$} & 1 & 20 & 94,45 & 1889 \\
\hline & 2 & 174 & 97,85 & 17026 \\
\hline \multirow{2}{*}{$\begin{array}{c}\text { Inovação } \\
(\mathrm{n}=194)\end{array}$} & 1 & 20 & 108,25 & 2165 \\
\hline & 2 & 174 & 96,26 & 16750 \\
\hline \multirow{2}{*}{$\begin{array}{c}\text { Condições de Pgto } \\
(\mathrm{n}=194)\end{array}$} & 1 & 20 & 103,8 & 2076 \\
\hline & 2 & 174 & 96,78 & 16839 \\
\hline \multirow{2}{*}{$\begin{array}{l}\text { Facilidade de Acesso } \\
\text { (n=194) }\end{array}$} & 1 & 20 & 99,45 & 1989 \\
\hline & 2 & 174 & 97,28 & 16926 \\
\hline \multirow{2}{*}{$\begin{array}{c}\text { Exposição PDV } \\
(\mathrm{n}=194)\end{array}$} & 1 & 20 & 103,53 & 2070,5 \\
\hline & 2 & 174 & 96,81 & 16844,5 \\
\hline
\end{tabular}

Legenda: 1,00 = Raramente + Periodicamente; 2,00 = Freqüentemente

Fonte: Dados da pesquisa (2013)

Como um dos objetivos específicos do estudo é verificar a correlação entre fatores que influenciam na imagem corporativa e a frequência de uso dos produtos, o primeiro teste estatístico realizado buscou verificar a correlação entre fatores de influência e a frequência de uso dos produtos. A Tabela 5 mostra os resultados da análise de correlação realizada por intermédio dos testes estatísticos não-paramétricos U de Mann-Whitney e Wilcoxon W. Os resultados apontam a ausência de correlação estatisticamente significativa entre as variáveis analisadas (fatores que influenciam na imagem corporativa e frequência de uso dos produtos), pois todos os fatores mensurados apresentaram valor de sig maior que 5\%; ou seja, $\mathrm{p}>0,05$. Assim, pode-se afirmar que, na opinião dos alunos universitários pesquisados, nenhum dos fatores que influenciam na imagem corporativa das empresas afeta a frequência de uso dos produtos, nem o fato de as empresas investirem em marketing social. Este resultado pode indicar que a frequência de uso dos produtos pode não estar linearmente correlacionada com os fatores que influenciam na imagem corporativa das empresas, mas com o conjunto de aspectos/atributos (sentimentos e associações percebidas) que constituem a imagem corporativa das empresas. 
Tabela 5 - Resultados dos testes estatísticos U de Mann-Whitney e Wilcoxon W

\begin{tabular}{|c|c|c|c|c|}
\hline \multirow{2}{*}{ Item } & Mann-Whitney U & Wilcoxon W & Z & P-value \\
\hline Serviço pós -venda & 1640,500 & 1850,500 &,- 400 &, 689 \\
\hline Variedade & 1694,000 & 1904,000 &,- 203 &, 839 \\
\hline Preço & 1706,000 & 1916,000 &,- 156 &, 876 \\
\hline Ética & 1730,000 & 1940,000 &,- 045 &, 964 \\
\hline Marketing social & 1682,500 & 1892,500 &,- 249 &, 803 \\
\hline Qualidade & 1679,000 & 1889,000 &,- 331 &, 741 \\
\hline Inovação & 1525,000 & 16750,000 &,- 944 &, 345 \\
\hline Condições de pgto & 1614,000 & 16839,000 &,- 547 &, 584 \\
\hline Facilidade de acesso & 1701,000 & 16926,000 &,- 174 &, 862 \\
\hline Exposição no PDV & 1619,500 & 16844,500 &,- 524 &, 600 \\
\hline
\end{tabular}

Variável de agrupamento: Frequência de uso.

Fonte: Dados da pesquisa (2013)

Para atender ao último objetivo específico desse estudo, o segundo teste estatístico buscou verificar a correlação entre os diferentes fatores que influenciam na imagem corporativa das empresas que investem em marketing social. A Tabela 6 exibe os resultados da análise de correlações ordinais baseada no Coeficiente de Correlação de Spearman (Siegel, 1975). Os resultados do teste estatístico mostram a existência de correlação significativa (sig menor que 5\%) entre marketing social (Csociais) e vários fatores de influência mensurados, quais sejam: serviços pós-venda $(\mathrm{p}=0,000)$, variedade de produtos $(\mathrm{p}=0,013)$, comportamento ético da empresa $(\mathrm{p}=0,000)$, qualidade dos produtos $(\mathrm{p}=0,003)$, inovação nos produtos $(\mathrm{p}=0,001)$, condições de pagamento $(\mathrm{p}=0,000)$, facilidades de acesso no ponto de venda $(\mathrm{p}=0,000)$ e exposição na mídia $(\mathrm{p}=0,000)$. 
Influência do Marketing Social na Imagem Corporativa

Tabela 6 - Resultados da análise de correlações de Spearman ( $\mathrm{n}=338)$

\begin{tabular}{|c|c|c|c|c|c|c|c|c|c|c|c|}
\hline & Serv & Varied & Prec & Etica & C. sociais & Qualid & Inov & Cond pg & Facilid & Expos & Percep \\
\hline \multirow[t]{3}{*}{ Serviço } & 1 & 0,232 & 0,346 & 0,299 & 0,233 & 0,344 & 0,285 & 0,354 & 0,319 & 0,214 & 0,12 \\
\hline & & 0 & 0 & 0 & 0 & 0 & 0 & 0 & 0 & 0,001 & 0,074 \\
\hline & 223 & 223 & 223 & 223 & 223 & 223 & 223 & 223 & 223 & 223 & 223 \\
\hline \multirow[t]{3}{*}{ Variedade } & 0,232 & 1 & 0,412 & 0,202 & 0,165 & 0,318 & 0,324 & 0,313 & 0,366 & 0,275 & 0,004 \\
\hline & 0 & , & 0 & 0,002 & 0,013 & 0 & 0 & 0 & 0 & 0 & 0,949 \\
\hline & 223 & 224 & 224 & 224 & 224 & 224 & 224 & 224 & 224 & 224 & 224 \\
\hline \multirow[t]{3}{*}{ Preço } & 0,346 & 0,412 & 1 & 0,274 & 0,112 & 0,485 & 0,327 & 0,393 & 0,318 & 0,183 & 0,045 \\
\hline & 0 & 0 & , & 0 & 0,095 & 0 & 0 & 0 & 0 & 0,006 & 0,504 \\
\hline & 223 & 224 & 224 & 224 & 224 & 224 & 224 & 224 & 224 & 224 & 224 \\
\hline \multirow[t]{3}{*}{ Ética } & 0,299 & 0,202 & 0,274 & 1 & 0,554 & 0,431 & 0,262 & 0,351 & 0,246 & 0,314 & 0,115 \\
\hline & 0 & 0,002 & 0 & , & 0 & 0 & 0 & 0 & 0 & 0 & 0,086 \\
\hline & 223 & 224 & 224 & 224 & 224 & 224 & 224 & 224 & 224 & 224 & 224 \\
\hline \multirow[t]{3}{*}{ Csociais } & 0,233 & 0,165 & 0,112 & 0,554 & 1 & 0,197 & 0,212 & 0,289 & 0,258 & 0,357 & 0,309 \\
\hline & 0 & 0,013 & 0,095 & 0 & , & 0,003 & 0,001 & 0 & 0 & 0 & 0 \\
\hline & 223 & 224 & 224 & 224 & 224 & 224 & 224 & 224 & 224 & 224 & 224 \\
\hline \multirow[t]{3}{*}{ Qualidade } & 0,344 & 0,318 & 0,485 & 0,431 & 0,197 & 1 & 0,542 & 0,319 & 0,392 & 0,177 & 0,06 \\
\hline & 0 & 0 & 0 & 0 & 0,003 & , & 0 & 0 & 0 & 0,008 & 0,368 \\
\hline & 223 & 224 & 224 & 224 & 224 & 224 & 224 & 224 & 224 & 224 & 224 \\
\hline \multirow[t]{3}{*}{ Inovação } & 0,285 & 0,324 & 0,327 & 0,262 & 0,212 & 0,542 & 1 & 0,465 & 0,383 & 0,352 & 0,107 \\
\hline & 0 & 0 & 0 & 0 & 0,001 & 0 & , & 0 & 0 & 0 & 0,11 \\
\hline & 223 & 224 & 224 & 224 & 224 & 224 & 224 & 224 & 224 & 224 & 224 \\
\hline \multirow[t]{3}{*}{ Cond Pg } & 0,354 & 0,313 & 0,393 & 0,351 & 0,289 & 0,319 & 0,465 & 1 & 0,517 & 0,375 & 0,166 \\
\hline & 0 & 0 & 0 & 0 & 0 & 0 & 0 & , & 0 & 0 & 0,013 \\
\hline & 223 & 224 & 224 & 224 & 224 & 224 & 224 & 224 & 224 & 224 & 224 \\
\hline \multirow[t]{3}{*}{ Facilidade } & 0,319 & 0,366 & 0,318 & 0,246 & 0,258 & 0,392 & 0,383 & 0,517 & 1 & 0,452 & 0,042 \\
\hline & 0 & 0 & 0 & 0 & 0 & 0 & 0 & 0 & , & 0 & 0,529 \\
\hline & 223 & 224 & 224 & 224 & 224 & 224 & 224 & 224 & 224 & 224 & 224 \\
\hline \multirow[t]{3}{*}{ Exposição } & 0,214 & 0,275 & 0,183 & 0,314 & 0,357 & 0,177 & 0,352 & 0,375 & 0,452 & 1 & 0,08 \\
\hline & 0,001 & 0 & 0,006 & 0 & 0 & 0,008 & 0 & 0 & 0 & , & 0,233 \\
\hline & 223 & 224 & 224 & 224 & 224 & 224 & 224 & 224 & 224 & 224 & 224 \\
\hline \multirow{3}{*}{$\begin{array}{l}\text { Percepção da } \\
\text { imagem } \\
\text { corporativa }\end{array}$} & 0,12 & 0,004 & 0,045 & 0,115 & 0,309 & 0,06 & 0,107 & 0,166 & 0,042 & 0,08 & 1 \\
\hline & 0,074 & 0,949 & 0,504 & 0,086 & 0 & 0,368 & 0,11 & 0,013 & 0,529 & 0,233 & , \\
\hline & 223 & 224 & 224 & 224 & 224 & 224 & 224 & 224 & 224 & 224 & 224 \\
\hline
\end{tabular}

Fonte: Dados da pesquisa (2013)

\subsection{Discussão dos resultados}

O propósito deste estudo é analisar em que medida campanhas de marketing social influenciam na imagem corporativa, na perspectiva do público alunos universitários. Os resultados desse estudo apontam que a grande maioria dos alunos universitários pesquisados conhece nomes de empresas que investem no Brasil em marketing social. Há vários nomes de empresas mencionados, principalmente organizações internacionais que atuam no Brasil no setor de bens de consumo, nas áreas de beleza, alimentação e saúde (ex: Nestlé, Procter \& Gamble e Grupo Fleury). Corroborando com o que já foi apontado por Pozo e Tachizawa (2012) e Froemming e Souza (2013), os resultados deste estudo contêm evidências de que o marketing social está presente no ambiente de negócios e pode ser utilizado por diferentes tipos de organização. A grande maioria dos alunos universitários pesquisados utiliza produtos de empresas que investem em marketing social, e grande parte deles utiliza com elevada frequência (pelo menos uma vez por semana) produtos dessas empresas, e costuma levar em consideração o fato de a marca investir em marketing social na decisão de compra de novos produtos. Como apontado por Nomura e
De Souza (2007), as empresas mencionadas pelos alunos pesquisados são de natureza privada ou pertencentes ao terceiro setor (ex: Fundação Bradesco).

Com relação aos fatores que influenciam na imagem corporativa, ao contrário do que foi afirmado por Lee e Kotler (2011), os resultados das análises realizadas no estudo indicam que marketing social é um fator de influência de baixa relevância na imagem corporativa, e que os fatores de influência mais importantes são qualidade e preços dos produtos ofertados. No estudo de Novais et al. (2010), o preço dos produtos foi também apontado como um importante fator de influência na decisão de compra, principalmente pelos alunos universitários de baixa renda. Cabe destacar, no entanto, que, no caso deste estudo, este resultado representa certa contradição na opinião dos alunos universitários pesquisados, pois parece incoerente afirmar que marketing social é um fator de influência com baixa relevância na imagem corporativa quando em questões anteriores (vide Tabelas 2 e 3) a grande maioria dos alunos pesquisados mencionou que utiliza produtos de empresas que investem em marketing social e leva em consideração a prática de marketing social das 
empresas quando da decisão de compra dos produtos.

Já diferente que foi apontado no estudo de Da Silva et al. (2012), envolvendo 104 alunos universitários na região metropolitana de Belo Horizonte, mostrando que o marketing social influencia no consumo dos produtos e que a maioria dos alunos universitários pesquisados $(54,8 \%$ da amostra) opta por adquirir produtos de empresas que apoiam causas sociais, os resultados dos testes estatísticos de correlação deste estudo apontam que não há qualquer correlação linear significativa entre os fatores que influenciam na imagem corporativa das empresas que investem em marketing social e a frequência de uso dos produtos pelos alunos universitários, nem mesmo em relação ao fator marketing social. Este resultado diverge também das conclusões do estudo de Novais et al. (2010), segundo o qual grande parte dos alunos universitários pesquisados mencionou adquirir produtos de empresas que investem em marketing social.

Corroborando com as afirmações de Lee e Kotler (2011), no entanto, neste estudo, os resultados do teste estatístico de correlação entre os diferentes fatores mensurados que influenciam na imagem corporativa das empresas apontaram que há correlação linear significativa entre marketing social e vários fatores que influenciam na imagem corporativa, quais sejam: serviços pós-venda, variedade de produtos, comportamento ético da empresa, qualidade dos produtos, inovação nos produtos, condições de pagamento, facilidades de acesso no ponto de venda e exposição na mídia. Assim, embora, no âmbito deste estudo, o marketing social tenha sido avaliado como um fator de influência com baixa relevância na imagem corporativa, pode-se supor que este tem certa influência indireta na imagem corporativa das empresas por intermédio de outros fatores de influência identificados neste estudo, e que a frequência de uso dos produtos pode não estar linearmente correlacionada com os fatores que influenciam na imagem corporativa, mas ao conjunto de aspectos/atributos que constituem a imagem corporativa das empresas que investem em marketing social. Estas suposições são hipóteses de pesquisa que carecem de serem testadas em futuros estudos acadêmicos sobre o tema.

\section{CONSIDERAÇÕES FINAIS}

Este estudo analisou em que medida campanhas de marketing social influenciam na imagem corporativa de empresas que investem em marketing social, na perspectiva do público alunos universitários. Para tanto, este estudo buscou: identificar as principais empresas que investem no Brasil em marketing social e a frequência de uso de seus produtos; levantar os fatores que influenciam na imagem corporativa das empresas que investem em marketing social; verificar a correlação entre fatores que influenciam na imagem corporativa e a frequência de uso dos produtos; a correlação entre os diferentes fatores que influenciam na imagem corporativa das empresas que investem em marketing social. Este estudo alcançou todos esses objetivos estabelecidos.

Sobre as empresas que investem em marketing social, este estudo revelou que a grande maioria dos alunos universitários pesquisados conhece nomes de empresas que investem no Brasil em marketing social e os nomes mais citados são Nestlé e Procter \& Gamble. A grande maioria dos alunos utiliza produtos das empresas citadas e costuma consumi-los frequentemente e, até por isso, leva em consideração o marketing social das empresas quando da decisão de compra dos produtos. Quanto aos fatores que influenciam na imagem corporativa, este estudo apontou que marketing social é um fator de influência com baixa relevância na opinião dos alunos universitários pesquisados, e que os fatores de influência mais importantes são qualidade e preços dos produtos. Os demais fatores mensurados foram apontados com moderada influência na imagem corporativa, incluindo exposição na mídia, ética nos negócios, serviço de pós-venda, variedade de produtos, inovação nos produtos, condições de pagamento e facilidade de acesso.

Com relação à correlação entre fatores que influenciam na imagem corporativa e frequência de uso dos produtos, os resultados desse estudo apontaram que não há correlação linear significativa, nem mesmo em relação ao marketing social. Sobre a correlação entre os diferentes fatores que influenciam na imagem corporativa, este estudo apontou que há correlação linear significativa entre marketing social e vários fatores mensurados que influenciam na imagem corporativa, quais sejam: serviços pós-venda, variedade de produtos, comportamento ético da empresa, qualidade dos produtos, inovação nos produtos, condições de pagamento, facilidades de acesso no ponto de venda e exposição na mídia.

Como contribuição teórica, este estudo representa uma ampliação do conhecimento acadêmico sobre influência do marketing social na imagem corporativa, um tema que ainda carece de estudos, como apontado por Nomura e De Souza (2007), Albino et al. (2013) e Higuchi e Vieira (2012). Os resultados contêm, também, evidências empíricas que podem auxiliar em futuros estudos 
sobre o tema, e possibilitar a identificação de novas hipóteses de pesquisa e direcionamentos para futuros estudos acadêmicos. Esta pesquisa contribuiu, ainda, para verificar proposições presentes na literatura, como fatores de influência na imagem corporativa citados por Lee e Kotler (2011), e resultados de estudos acadêmicos, como as pesquisas de Novais et al. (2010) sobre a importância do preço na decisão de compra de alunos universitários, de Da Silva et al. (2012) sobre correlação entre fatores de influência e a frequência de uso de produtos, e de Pozo e Tachizawa (2012) sobre a presença do marketing social no ambiente de negócios.

Em termos de limitações de estudo, cabe destacar que a pesquisa de campo adotou amostragem não-probabilística (com seleção por conveniência dos elementos amostrais), sendo assim seus resultados não são conclusivos e não podem ser generalizados para a população desse estudo. A avaliação da influência do marketing social na imagem corporativa pode também necessitar a mensuração de outras variáveis de pesquisa não consideradas neste estudo, como identidade da marca, posicionamento da marca, personalidade da marca e comunicação de marketing da marca. Diante disso, como direcionamento para futuros estudos, sugere-se a reaplicação deste estudo em outros contextos, seja em outras universidades do Estado de São Paulo ou em outros estados do Brasil. Há necessidade de pesquisas com emprego de processo de amostragem aleatório e com a aplicação de métodos qualitativos para compreender melhor os resultados deste estudo, e estudos com desenvolvimento de modelos teóricos conceituais que expliquem melhor o fenômeno investigado.

Novos estudos acadêmicos são também necessários para elucidar a questão/hipótese sobre a influência indireta do marketing social na imagem corporativa, por intermédio de outros fatores identificados neste estudo, e sobre a correlação entre frequência de uso dos produtos com o conjunto de aspectos/atributos que constituem a imagem corporativa das empresas que investem em marketing social.

\section{REFERÊNCIAS}

Albino, A. et al. (2013) Responsabilidade social corporativa e uso de iniciativas de marketing para o bem-estar da sociedade: estudo no APL Moveleiro de Ubá. Revista de Administração da UEG, 3 (2), 43.
Andreasen, B., \& Kotler, P. (1991) A framework for marketing image management. Sloan management review, 32(2), 94-104.

Bloom, P., Hoeffler, S., Keller, K., \& Meza, C. (2012) How social-cause marketing affects consumer perceptions image. Recuperado em: 15 març.2015, de: http://sloanreview.mit.edu/article/howsocialcause-marketing-affects-consumerperceptions/.

Brenkert, G. (2002) Ethical challenges of social marketing. Journal of Public Policy and Marketing, Chicago, Spring.

Brunk, K. (2010) Exploring origins of ethical company/brand perceptions: a consumer perspective of corporate ethics. Journal of Business Research, 63(3), 255-262.

Camargo, M. (2001) Gestão do terceiro setor no Brasil. São Paulo: Futura.

Carrol, A., \& Buchholtz, A. (2014) Business and society: ethics, sustainability, and stakeholder management. London: Cengage Learning.

Collis, J, \& Hussey, R. (2005) Pesquisa em administração: um guia prático para alunos de graduação e pós-graduação. Porto Alegre: Bookman.

Da Silva, F. et al. (2012) Análise das estratégias de marketing associadas a causas sociais: um estudo na cidade de Belo Horizonte. Revista da Faculdade de Administração e Economia, 3 (2), 32-55.

De Oliveira, F. (2006) Estratégias de responsabilidade social corporativa: um estudo sobre os 231 casos concretos do Instituto Ethos. Recadm, 5 (1), 1-14.

Froemming, L., \& Souza, N. (2013) A responsabilidade social corporativa e a importância do marketing social. Revista de Administração, 8 (15), 69-84.

Grönroos, C. (1995) Marketing: gerenciamento de serviços: a competição por serviços na hora da verdade. Rio de Janeiro: Campus.

Hair, J., Black, B., Babin, B., Anderson, R., \& Tatham, R. (2009) Análise multivariada de dados. Porto Alegre: Bookman. 
Higuchi, A., \& Vieira, F. (2012) Responsabilidade social corporativa e marketing social corporativo: uma proposta de fronteira entre estes dois conceitos. Acta Scientiarum. Human and Social Sciences, 34 (1), 31-40.

Ikeda, A., Campomar, M., \& Miuri, I. (2000) Combate ao desperdício da energia elétrica: um caso de marketing societal. Revista de Administração da Faculdade de Economia e Contabilidade da USP, 35 (1), 5-7.

Instituto Ethos de Empresas e Responsabilidade Social [Ethos] (2015). Recuperado em: 15 març.2015, de:

http://fenix.ethos.org.br/ListaAssociadasPorPort eEstado.aspx .

Kloppenborg, T. J., \& Tesch, D. (2015) How executive sponsors influence project success. MIT Sloan Management Review, 56(3), 27.

Kotler, P., \& Fox, K. (1994) Marketing estratégico para instituições educacionais. São Paulo: Atlas.

Kotler, P. (1978) Marketing para as organizações que não visam lucro. São Paulo: Atlas.

Kotler, P., \& Roberto, E. (1992) Marketing social: estratégias para alterar o comportamento do público. Rio de Janeiro: Campus.

Lee, N., \& Kotler, P. (2011) Social marketing: Influencing behaviors for good. New York: Sage.

Maon, F., Lindgreen, A., \& Swaen, V. (2010) Organizational stages and cultural phases: a critical review and a consolidative model of corporate social responsibility development. International Journal of Management Reviews, 12(1), 20-38.

Mazutis, D., \& Slawinski, N. (2015) Reconnecting business and society: perceptions of authenticity in corporate social responsibility. Journal of Business Ethics, 131(1), 137-150

Mejri, M., \& De Wolf, D. (2012) Analysis of retailers' communication approaches in sustainability and social responsibility reports. International Journal of Marketing Studies, 4(2), 30-44.
Nascimento, C., Jimenez, G., \& Campomar, M. (2014) A rede social como fonte de informação para o processo de decisão de compra. Future Studies Research Journal: Trends and Strategies, 6(2), 30-47.

Nomura, J., De Souza, M. (2007) A critic revision of the social marketing concept. Iberoamerican Journal of Strategic Management (IJSM), 3 (1), 45-52.

Novais, F. et al. (2010) Marketing relacionado as causas como vantagem competitiva: um estudo com jovens universitários na cidade de Belo Horizonte. Semead-Seminários em Administração, (13).

Moretti, S., \& Figueiredo, J. (2008) Análise bibliométrica da produção sobre responsabilidade social das empresas no Enanpad: evidências de um discurso monológico Revista de Gestão Social e Ambiental, 1(3), 21-38.

Patino, A., Kaltcheva, V., Pitta, D., Sriram, V., \& Winsor, R. (2014) How important are different socially responsible marketing practices? An exploratory study of gender, race, and income differences. Journal of Consumer Marketing, 31(1), 2-12.

Pozo, H., \& Tachizawa, T. (2012) Responsabilidade social corporativa e marketing social: um estudo exploratório em empresas para o fortalecimento do turismo na região da baixada santista (São Paulo/BR). Guia de Estilo para Autores, (10), 357.

Pringle, H., \& Thompson, M. (2000) Marketing social: marketing para causas sociais e a construção das marcas. São Paulo: Makron Books.

Rabaça, C., \& Barbosa, A. (1996) Marketing: segredos e estratégias. São Paulo: Saraiva.

Richardson, R. (2008) Pesquisa social: métodos e técnicas. São Paulo: Atlas.

Siegel, S. (1975) Estatística não-paramétrica para as ciências do comportamento. São Paulo: McGraw-Hill do Brasil.

Silva, R., \& Chauvel, M. (2011) A responsabilidade social das empresas e seus efeitos no comportamento do consumidor: um 
estudo qualitativo. Revista de Gestão Social e Ambiental, 5(2), 172-186.

Silva, R., \& Motta, P. (2014) Os efeitos do causerelated marketing no comportamento do consumidor. Revista Pensamento Contemporâneo em Administração, 8(2), 18-34.

Tavares, M. (1998) A força da marca. São Paulo: Harbra.

Vieira, F., Higuchi, A., Schneider-de-Oliveira, R., \& Corrêa, P. (2007) Marketing social corporativo: estado-da-arte e proposição de um modelo conceitual. Associação Nacional de Pós-graduação e Pesquisa em Administração, 31, 1-6.
Wilmer, W. \& Basi, D. (2007). Social marketing: advances in research and theory. Journal of Non-Profit \& Public Sector Marketing, 17, 1-2.

Weinreich, N. (2011) Hands-on social marketing: a step-by-step guide to designing change for good. Sage Publications Inc.

Wu, S., \& Wang, W. (2014) Impact of CSR perception on brand image, brand attitude and buying willingness: a study of a global café. International Journal of Marketing Studies, 6(6), 43. 